\title{
Cardiac hypertrophy, low blood pressure, and low aldosterone levels in mice devoid of the three circadian PAR bZip transcription factors DBP, HLF, and TEF
}

Running head: Cardiac dysfunction in PAR bZip deficient mice

Qing Wang ${ }^{1,4}$, Marc Maillard ${ }^{1}$, Ueli Schibler ${ }^{2}$, Michel Burnier ${ }^{1}$, and Frédéric Gachon ${ }^{2,3}$

${ }^{1}$ Service of Nephrology and Hypertension, CHUV, Lausanne, CH-1011, Switzerland

${ }^{2}$ Department of Molecular Biology \& National Center of Competence Research (NCCR)

Frontiers in Genetics, University of Geneva, Geneva, CH-1211, Switzerland

${ }^{3}$ Department of Pharmacology and Toxicology, University of Lausanne, Lausanne, CH-1005,

Switzerland

${ }^{4}$ Huazhong University of Science and Technology, Wuhan, China

Correspondence should be addressed to

Frédéric Gachon

University of Lausanne

Department of Pharmacology and Toxicology

Rue du Bugnon, 27

CH-1005 Lausanne

Switzerland

Tel: (00 41) 216925364

Fax: (00 41) 216925355

E-mail: Frederic.Gachon@unil.ch 


\begin{abstract}
The cardiovascular system is under the control of the circadian clock, and disturbed circadian rhythms can induce cardiovascular pathologies. This cyclic regulation is probably brought about by the circadian expression of genes encoding enzymes and regulators involved in cardiovascular functions. We have previously shown that the rhythmic transcription of output genes is, in part, regulated by the clock-controlled PAR bZip transcription factors DBP (albumin D-element Binding Protein), HLF (Hepatic Leukemia Factor), and TEF (Thyrotroph Embryonic Factor). The simultaneous deletion of all three PAR bZip transcription factors leads to increased morbidity and shortened life span. Here, we demonstrate that $\mathrm{Dbp} / \mathrm{Tef} / \mathrm{Hlf}$ triple knockout mice develop cardiac hypertrophy and left ventricular dysfunction associated with a low blood pressure. These dysfunctions are exacerbated by an abnormal response to this low blood pressure characterized by low aldosterone levels. The phenotype of PAR bZip knockout mice highlights the importance of circadian regulators in the modulation of cardiovascular functions.
\end{abstract}

Keywords: circadian clock, PAR bZip transcription factors, cardiac physiology, blood pressure, kidney function 


\section{Introduction}

Circadian clocks are allowing a large variety of organisms to adapt their physiology and behaviour to environmental changes in a proactive fashion. In mammals, most aspects of physiology, including the regulation of the cardiovascular system, are under the control of this molecular timekeeping system (13). Indeed, blood pressure and heart rate are subject to circadian oscillations, which can influence both the degree and the type of cardiac hypertrophy and remodeling when they are disturbed $(2,26)$. In addition, a misalignment between the intrinsic circadian period and the environmentally imposed rhythms can induce cardiovascular disease $(16,17,19)$. Hence, cardiovascular failures, such as acute myocardial infarction, are more frequent at certain hours of the day $(15,27)$. Centrally mediated autonomic or neurohumoral activation plays an important role in the development of these circadian variations. However, tissue- or cell-autonomous pathways imposed by local peripheral clocks may also contribute to these rhythmic patterns.

In mammals, the master pacemaker resides in the suprachiasmatic nuclei (SCN) of the hypothalamus. SCN neurons, whose circadian phase is daily reset by light-dark cycles, synchronize peripheral circadian clocks through both synaptic connections and humoral signals $(11,21)$. Nevertheless, feeding-fasting rhythms, which are themselves imposed by SCN-driven rest-activity cycles, are the dominant Zeitgebers for most peripheral organs, including heart, liver, and kidney $(3,20)$. At the molecular level, the circadian clockwork circuitry is thought to be based on transcriptional-translational negative feedback loops, involving the transcriptional activators BMAL1 and its heterodimerization partners CLOCK and NPAS2, and the negatively acting proteins PERIOD 1 (PER1), PERIOD 2 (PER2), CRYPTOCHROME 1 (CRY1), and CRYPTOCHROME 2 (CRY2) (12). These core clock components regulate a large number of output genes, either directly or by driving the rhythmic expression of downstream transcription factors, such as DBP (albumin D-site- 
Binding Protein), HLF (Hepatic Leukemia Factor), and TEF (Thyrotroph Embryonic Factor), the three members of the PAR bZip (Proline and Acidic amino acid-Rich basic leucine Zipper) protein family (8).

Previous studies have shown that the simultaneous loss of Dbp, Hlf, and Tef in PAR bZip triple knockout (KO) mice leads to an increased morbidity and severely shortens the life span of these animals (10). Thus, about $50 \%$ of young adults succumb to spontaneous and sound-induced seizures (10). After three months of age, the surviving animals become more resilient to epileptic attacks, but they display symptoms of accelerated ageing. At least in part, a deficiency in xenobiotic detoxification might account for these symptoms, as PAR bZip transcription factors are important regulators of genes implicated in this process (9). Here, we present evidence that these mice also display a cardiac hypertrophy. To gain insight into the potential roles of PAR bZip transcription factors in cardiovascular functions, we investigated cardiovascular phenotypes of $\mathrm{Dbp}^{-/-} / \mathrm{Hlf}^{-/} / \mathrm{Tef}^{-/-}$triple $\mathrm{KO}$ mice (henceforth called KO mice) in greater detail.

\section{Materials and Methods}

\section{Animals}

PAR bZip triple knockout mice have been previously described (10). Four to eight month-old male mice, 72 in total, were used in all studies. Four different groups of mice were used in the different protocols as follows: 30 mice (10 of each genotype) were used for the analysis of ENaC activity and urinary electrolytes, left ventricular hemodynamic measurements, and plasma aldosterone concentrations; 15 mice ( 5 of each genotype) were used for the telemetric measurements of blood pressure and heart rate; 15 mice ( 5 of each genotype) were used for determining the dose-response to $\beta 1$-receptor blockade; and 12 mice (6 of each genotype) 
were used for measuring the response of $\mathrm{ENaC}$ activity to exogenous aldosterone. Experiments on live animals were approved by an institutional committee for the humane use of animals. All mice were housed in a temperature- and humidity-controlled room with an automatic $12 \mathrm{~h}$ light/12 $\mathrm{h}$ dark cycle and fed ad libitum with a regular chow diet containing 3 $\mathrm{mg} \mathrm{Na}^{+} / \mathrm{g}$ of food.

\section{Cardiovascular and renal parameters:}

Blood pressure (BP) was measured by telemetry. For this purpose, mice were first anaesthetized with $1-2 \%$ halothane mixed with oxygen. A catheter tip of TA11PA-C10 BP transmitter (Data Sciences International, St. Paul, MN, USA) was inserted into the aortic arch via left carotid artery. The body of the transmitter was implanted subcutaneously on the right side of the chest. After a 2-week recovery period, BP and heart rate (HR) of the free moving animals were monitored continuously with a scheduled sampling (10 seconds every 5 minutes) for two weeks, using a computerized data-acquisition system (Dataquest ART software version 3.1) provided by Data Science International.

Cardiac haemodynamic parameters were also measured in conscious mice, using a fluid-filled catheter with high frequency response and high fidelity enabling to monitor left ventricular hemodynamic parameters in non-anesthetized mice, as described previously (22).

The activity of the epithelial sodium channel $(\mathrm{ENaC})$ in the distal colon was measured in vivo as the amiloride-sensitive rectal potential difference (ami-s-PD or $\triangle \mathrm{PD}_{\text {amil }}$ ) as described previously (23). To test whether the $\mathrm{ENaC}$ channel was normally induced by aldosterone, rectal $\Delta \mathrm{PD}_{\text {amil }}$ was measured before and 6 hours after administration of aldosterone $(6 \mathrm{mg} / \mathrm{kg}$, i.p).

Urine samples were collected into $1.5 \mathrm{~mL}$ Eppendorf tubes for $4 \mathrm{~h}$ at the same Zeitgeber time as rectal $\mathrm{PD}$ measurements were taken. Serum and urinary $\mathrm{Na}^{+}, \mathrm{K}^{+}$, and creatinine 
concentrations were analyzed by flame photometry (model 943, Instrumentation Laboratory). Plasma aldosterone was measured by conventional radioimmunoassays.

The activity of the sympathetic nervous system in these mice was evaluated by measuring the cardiac and heart rate response to selective $\beta_{1}$-adrenoreceptor blockade using the beta-blocker atenolol. Atenolol was administered intravenously at increasing doses $(3,10$, and $60 \mathrm{mg} / \mathrm{kg})$, and $\mathrm{BP}$, heart rate, and left ventricular function was monitored in conscious mice.

\section{Statistical analysis}

All physiological parameters are presented as mean \pm SEM. Statistical comparisons between groups were performed by a 1-way ANOVA, followed by a Newman-Keuls test. $P \leq 0.05$ was considered the minimal level of significance.

\section{Results}

\section{PAR bZip deficient mice develop cardiac hypertrophy and left ventricular dysfunction}

Examination of PAR bZip KO mice revealed a cardiac hypertrophy. Indeed, as compared to wild-type (WT) and heterozygous (HZ) animals, the heart weight over body weight (HW/BW) ratio was significantly increased in $\mathrm{KO}$ mice (Figure 1A), even if the histopathological analysis revealed no abnormality (data not shown). These animals also displayed characteristics of both systolic and diastolic dysfunction in the left ventricle, including a lower left ventricular systolic pressure (LVSP), an increase in left ventricular end-diastolic pressure (LVEDP), a prolongation of the left ventricular relaxation time constant (Weiss Tau), and a significant decrease in the absolute values of $\mathrm{LV} d P / d t_{\text {min }}$ and $\mathrm{LV} d P / d t_{\max }$ (Figure 1C-F). Moreover, the lung weight (LW) to BW ratio was increased in $\mathrm{KO}$ mice, indicating increased pulmonary water content due to left ventricular dysfunction (Figure 1B).

\section{PAR bZip deficient mice display altered blood pressure and heart rate}

Cardiac hypertrophy has multiple causes, including arterial hypertension. We thus measured by telemetry the blood pressure (BP) and heart rate (HR) of these mice over several days to 
analyze the diurnal variations of these parameters. As shown in Figure 2, diurnal rhythms of $\mathrm{BP}$ and $\mathrm{HR}$ were maintained in $\mathrm{KO}$ as well as in $\mathrm{HZ}$ mice, with dipping during the day then rising during the night. However, $\mathrm{BP}$ was significantly lower and $\mathrm{HR}$ higher in $\mathrm{KO}$ mice than in WT mice. HZ mice had an intermediate phenotype, with an increased heart rate and a slightly (but not statistically significant) lower BP than WT mice.

\section{PAR bZip deficient mice have an increased activity of the sympathetic nervous system}

As mentioned above, both $\mathrm{KO}$ and $\mathrm{HZ}$ mice had an increased heart rate when compared to WT mice. This may reflect an increased sympathetic activity in order to maintain cardiac output. To test this hypothesis, we investigated the activity of the sympathetic nervous system in these mice by measuring the cardiac and heart rate response to selective $\beta_{1}$-adrenoreceptor blockade using the drug atenolol. Atenolol was administered intravenously at increasing doses, and BP, HR, and left ventricular function were monitored in conscious mice by invasive catheterization. As shown in Figure 3, the responsiveness to beta-receptor blockade was significantly enhanced for all parameters in $\mathrm{KO}$ mice, with an intermediate response in $\mathrm{HZ}$ animals. This result confirmed that $\mathrm{KO}$ mice, and to a lesser extend $\mathrm{HZ}$ mice, presented an increased sympathetic activity. This led to an accelerated HR, probably to compensate for the low cardiac output.

\section{PAR bZip deficient mice display an inappropriate response to low blood pressure}

In response to a decreased blood pressure, the kidney normally increases salt re-absorption. We thus evaluated salt absorption by measuring the difference in salt excretion with the urine over several days. Urine samples were collected in the morning (ZT0 to ZT4; ZT is Zeitgeber Time; ZT0 and ZT12, are the times when light is turned on and off, respectively) and during the night (ZT12 to ZT16). Urinary volumes were significantly greater in $\mathrm{KO}$ than in $\mathrm{HZ}$ and 
WT mice, like water and sodium (food) intakes (Figure 4A-C). However, body weight and serum sodium and potassium concentrations were comparable among the three groups (data not shown). While urinary excretion of creatinine was similar in the three groups of mice, sodium excretion, expressed as sodium to creatinine ratio $(\mathrm{Na} / \mathrm{Cre})$, and, to a lesser extent, potassium excretion $(\mathrm{K} / \mathrm{Cre})$ were higher in $\mathrm{KO}$ than in $\mathrm{HZ}$ and $\mathrm{WT}$ mice over the course of the day (Figure 4D-F). This increased sodium excretion was probably caused by the augmented salt absorption. However, contrary to what would have been predicted, salt reabsorption was not increased in these mice as a result of low blood pressure.

Indeed, we expected an increase in salt re-absorption through the activation of the amiloride-sensitive epithelial sodium channel $(\mathrm{ENaC})$, the main regulator of salt reabsorption located in the distal nephron of the kidney. This is normally achieved by rising the activity of the renin-angiotensin-aldosterone system. The absence of increased salt reabsorption in the mutant mice could therefore be the consequence of an inappropriate activation of the renin-angiotensin-aldosterone system. The triple KO mice have a mixed C57Bl/6-129sv-129Ola genetic background and their haploid genome harbors two Renin genes. As described previously, the presence of the Renin2 gene causes a 100-fold increase in the plasma renin concentration, which masked the usual negative feedback control on renin activity (24). However, when we measured Renin gene mRNA accumulation in the kidney of KO mice we observed a 1.6 fold reduction in Renin1 mRNA accumulation in response to low blood pressure, rather than the expected increase (data not shown). We also wished to evaluate the adaptation of the aldosterone system to low blood pressure in $\mathrm{KO}$ mice. To this end we determined the acute response of the $\mathrm{ENaC}$ channel to exogenous aldosterone in the distal colon. This tissue is a secondary site of $\mathrm{ENaC}$ expression that is more accessible than nephrons and permits the direct determination of the channel activity by monitoring the net change in rectal ami-s-PD. As shown in Figure 5A, the ENaC activity was not significantly 
different in $\mathrm{KO}$ and WT mice in response to exogenous aldosterone, suggesting that the intrinsic channel responsiveness was not affected in PAR bZip KO mice. However, plasma aldosterone levels were reduced by approximately $40 \%$ in $\mathrm{KO}$ mice compared to WT and $\mathrm{HZ}$ mice (Figure 5B), reflecting an inappropriate response to low blood pressure.

To investigate the possible consequence of the low aldosterone levels on the diurnal $\mathrm{ENaC}$ activity in vivo, we monitored the amiloride-sensitive potential difference (ami-s-PD) in the distal colon. The rectal ami-s-PD of both WT and HZ mice followed a clear diurnal rhythm, with a low activity in the morning (resting phase) and a higher activity at night (activity phase) (Figure 6). In contrast, the diurnal rhythm of ami-s-PD was completely abolished in PAR bZip deficient mice, suggesting an impaired rhythmic activity of the amiloride sensitive channel and confirming the inappropriate response to low blood pressure.

\section{Discussion}

The PAR bZip transcription factors DBP, HLF, and TEF serve as mediators in translating daily oscillations generated by the molecular clockwork circuitry into overt circadian rhythms in gene expression and physiology. The observation that $\mathrm{KO}$ mice display cardiac hypertrophy motivated us to examine their role in the cardiovascular system. Our results demonstrated that the deletion of PAR bZip transcription factors had a major impact on cardiac and renal functions, leading to the development of cardiac hypertrophy, left ventricular dysfunction, and increased sympathetic activity. Unexpectedly these symptoms were associated with low blood pressure and low aldosterone levels, possibly caused by an inappropriate response to this state.

As mentioned previously, a low BP and an increased HR could also be a manifestation of a cardiac dysfunction. Despite the low BP, KO mice had a clear left ventricular 
hypertrophy. Moreover, KO mice displayed several hemodynamic characteristics of heart failure, including a lower left ventricular systolic pressure, an increase in left ventricular enddiastolic pressure, a prolongation of the left ventricular relaxation time constant (Weiss Tau), and a significant decrease in the absolute values of $\mathrm{LV} d P / d t_{\min }$ and $\mathrm{LV} d P / d t_{\max }$. In addition, the lung weight to body weight ratio was enlarged, suggesting an increased pulmonary water content. Moreover, KO mice exhibited an enhanced hemodynamic response to acute $\beta_{1^{-}}$ receptor blockade when compared to $\mathrm{WT}$ or $\mathrm{HZ}$ mice, demonstrating an increased activity of the sympathetic nervous system commonly observed early in the course of diastolic dysfunction (6). These results indicated that the deletion of PAR bZip transcription factors had a major impact on cardiac function, which appeared to be gene dosage dependent. In fact HZ mice had an intermediate phenotype with some signs of compensated diastolic dysfunction.

The normal response to low blood pressure suggested an increase in aldosterone synthesis. However, this was not the case in PAR bZip KO mice, as these animals displayed a reduced plasma aldosterone level. Aldosterone synthesis is achieved by the processing of corticosterone by aldosterone synthase. Interestingly, it had been shown previously that corticosterone synthesis is controlled by the circadian clock, which regulates, directly or indirectly, the expression of enzymes involved in corticosterone synthesis in the adrenal gland (18). In addition, it has recently been demonstrated that the circadian clock, through the PAR bZip transcription factors, also modulates the expression of HSD3B6, an enzyme directly involved in aldosterone synthesis in the adrenal gland (5). It is thus conceivable that the PAR bZip transcription factors could be implicated in this process, potentially explaining the lower levels of aldosterone in $\mathrm{KO}$ mice.

PAR bZip deficient mice also exhibited an increased urinary sodium and potassium excretion. It is difficult to conclude in a decisive manner whether the increased urinary sodium excretion was a primary consequence of PAR bZip loss-of-function or just a result of increased sodium intake. To address this question, other manifestations of an impaired renal 
regulation of sodium excretion were explored. The amiloride-sensitive epithelial sodium channel $(\mathrm{ENaC})$ located in the distal nephron and distal colon is responsible for the fine regulation of sodium excretion (14). In contrast to WT and $\mathrm{HZ}$ mice, $\mathrm{ENaC}$ activity in the distal colon was very low in KO mice. Moreover, the daily rhythm of ami-s-PD was completely abolished in $\mathrm{KO}$ mice. The low activity and the absence of a clear diurnal rhythm of $\mathrm{ENaC}$ could not be explained by a higher salt intake, as previous experiments conducted in mice receiving a very high sodium intake ( $16 \mathrm{mg} \mathrm{Na}^{+} / \mathrm{g}$ of food) have shown that the rhythm is maintained even at lower levels of $\mathrm{ENaC}$ activity (23). This suggested that the low aldosterone synthesis in PARb Zip KO mice contributed to the inappropriate response to low blood pressure.

The lower BP of KO mice was associated with an increased HR, which likely reflected a compensatory mechanism to maintain cardiac output through the persistent activation of the sympathetic nervous system, which could lead to cardiac hypertrophy. The cardiac dysfunction could therefore be the consequence of the persistently high HR (7). Indeed, a relative hypovolemia due for example to a sodium loss could explain the cardiac hypertrophy, and the renal sodium excretion was, in fact, significantly higher in KO mice than in WT animals, despite of the low BP. However, it is also conceivable that the loss of PAR bZip transcription factors engendered a primary cardiac dysfunction. While left ventricular dysfunction was the main cause of the low BP, one would have expected a lower sodium excretion in $\mathrm{KO}$ mice, due to the renal hypoperfusion associated with a low cardiac output. However, in that case the renin-angiotensin-aldosterone system should have been activated, which was clearly not observed in PAR bZip deficient mice. This suggested that the phenotype was probably caused by the combination of a perturbed heart function and an inappropriate response of the renin-angiotensin-aldosterone system to low blood pressure, which contributed to a defective salt re-absorption in the kidney. This observation supported 
the hypothesis of a perturbed kidney function, which could have been caused by a deregulated expression of genes involved in $\mathrm{BP}$ regulation in these mice, as reported by Zuber A.M. et al. (28).

Clock-controlled transcription factors, such as the PAR bZip proteins, govern the rhythmic expression of genes and hence contribute to the regulation of several physiological rhythms (8). Interestingly, in contrast to the observations made in mice with a disruption of the gene encoding BMAL1, the master regulator of the molecular circadian clock (1), our results showed that the circadian pattern of blood pressure was maintained in $\mathrm{KO}$ mice. Nevertheless, BP was significantly lower in KO mice (and to a lesser degree in $\mathrm{HZ}$ mice), an observation indicating that important determinants of the regulation of blood pressure were affected by the loss of the PAR bZip transcription factors. Interestingly, a lower BP with a conserved diurnal rhythm was also found in Npas2 (1) and Clock (28) KO mice, the genes specifying the heterodimerization partner of BMAL1. These two animal models, similar to PAR bZip KO mice, display a normal diurnal rhythm of locomotor activity with a decreased expression of the output genes regulated by the clock (4). The fact that these three mouse models exhibit a similar BP phenotype suggests that the cyclic expression of relevant clock outputs genes, rather than a rhythmic behavior, is required to regulate the level of BP. Unfortunately, the cardiac phenotypes of these mice have not yet been studied in detail.

\section{Perspectives and Significance}

In humans, a recent study, aimed at inducing a circadian misalignment in ten adults by submitting them to 28 hours "days", showed that already after eight days some subjects displayed decreased leptin levels, increased blood glucose concentrations (despite increased insulin levels), phase-inverted daily cortisol rhythms, increased mean arterial pressure, and reduced sleep efficiency (19). Moreover, two different haplotypes of the BMall gene have 
been linked to a susceptibility to hypertension and type 2 diabetes (25). Given the extraordinary phylogenetic conservation of the BMAL1-regulated PAR bZip transcription factors $(8,9)$, we consider likely that these circadian regulators could also be involved in the modulation of cardiovascular functions in humans.

\section{Acknowledgments}

We are very grateful to Dmitry Firsov and Olivier Bonny (University of Lausanne) for critical reading of the manuscript.

\section{Grants}

This work was supported by the Hypertension Research Foundation to M.B.; the Swiss National Foundation (through an individual research grants to M.B., U.S., and F.G. and the National Center of Competence in Research Program Frontiers in Genetics to U.S.); the Canton of Geneva (U.S.), the Bonizzi-Theler Stiftung (U.S.), the 6th European Framework Project EUCLOCK (U.S.); the Institut National de la Santé et de la Recherché Médicale (F.G), and the Canton of Vaud (F.G. and M.B).

\section{References}

1. Curtis AM, Cheng Y, Kapoor S, Reilly D, Price TS, and FitzGerald GA. Circadian variation of blood pressure and the vascular response to asynchronous stress. Proc Natl Acad Sci U S A 104: 3450-3455, 2007.

2. Cuspidi C, Lonati L, Sampieri L, Macca G, Valagussa L, Zaro T, Michev I, Fusi V, Leonetti G, and Zanchetti A. Impact of nocturnal fall in blood pressure on early cardiovascular changes in essential hypertension. J Hypertens 17: 1339-1344, 1999. 
3. Damiola F, Le Minh N, Preitner N, Kornmann B, Fleury-Olela F, and Schibler U. Restricted feeding uncouples circadian oscillators in peripheral tissues from the central pacemaker in the suprachiasmatic nucleus. Genes Dev 14: 2950-2961, 2000.

4. DeBruyne JP, Noton E, Lambert CM, Maywood ES, Weaver DR, and Reppert SM. A Clock Shock: Mouse CLOCK Is Not Required for Circadian Oscillator Function. Neuron 50: 465-477, 2006.

5. Doi M, Takahashi Y, Komatsu R, Yamazaki F, Yamada H, Haraguchi S, Emoto N, Okuno Y, Tsujimoto G, Kanematsu A, Ogawa O, Todo T, Tsutsui K, van der Horst GTJ, and Okamura H. Salt-sensitive hypertension in circadian clock-deficient Cry-null mice involves dysregulated adrenal Hsd3b6. Nat Med 16: 67-74, 2010.

6. Dorn GW 2nd. Adrenergic pathways and left ventricular remodeling. J Card Fail 8: S370-S373, 2002.

7. Dorn GW 2nd and Hahn HS. Genetic Factors in Cardiac Hypertrophy. Ann N Y Acad Sci 1015: 225-237, 2004.

8. Gachon F. Physiological function of PARbZip circadian clock-controlled transcription factors. Ann Med 39: 562 - 571, 2007.

9. Gachon F, Fleury Olela F, Schaad O, Descombes P, and Schibler U. The circadian PAR-domain basic leucine zipper transcription factors DBP, TEF, and HLF modulate basal and inducible xenobiotic detoxification. Cell Metab 4: 25-36, 2006.

10. Gachon F, Fonjallaz P, Damiola F, Gos P, Kodama T, Zakany J, Duboule D, Petit B, Tafti M, and Schibler U. The loss of circadian PAR bZip transcription factors results in epilepsy. Genes Dev 18: 1397-1412, 2004.

11. Gachon F, Nagoshi E, Brown SA, Ripperger J, and Schibler U. The mammalian circadian timing system: from gene expression to physiology. Chromosoma 113: 103-112, 2004. 
12. Gallego M and Virshup DM. Post-translational modifications regulate the ticking of the circadian clock. Nat Rev Mol Cell Biol 8: 139-148, 2007.

13. Green CB, Takahashi JS, and Bass J. The Meter of Metabolism. Cell 134: 728-742, 2008.

14. Hummler E and Rossier BC. Physiological and pathophysiological role of the epithelial sodium channel in the control of blood pressure. Kidney Blood Press Res 19: 160-165, 1996.

15. Lemmer B. The importance of circadian rhythms on drug response in hypertension and coronary heart disease--from mice and man. Pharmacol Ther 111: 629-651, 2006.

16. Martino TA, Oudit GY, Herzenberg AM, Tata N, Koletar MM, Kabir GM, Belsham DD, Backx PH, Ralph MR, and Sole MJ. Circadian rhythm disorganization produces profound cardiovascular and renal disease in hamsters. Am J Physiol Regul Integr Comp Physiol 294: R1675-1683, 2008.

17. Martino TA, Tata N, Belsham DD, Chalmers J, Straume M, Lee P, Pribiag H, Khaper N, Liu PP, Dawood F, Backx PH, Ralph MR, and Sole MJ. Disturbed Diurnal Rhythm Alters Gene Expression and Exacerbates Cardiovascular Disease With Rescue by Resynchronization. Hypertension 49: 1104-1113, 2007.

18. Oster H, Damerow S, Kiessling S, Jakubcakova V, Abraham D, Tian J, Hoffmann MW, and Eichele G. The circadian rhythm of glucocorticoids is regulated by a gating mechanism residing in the adrenal cortical clock. Cell Metab 4: 163-173, 2006.

19. Scheer FAJL, Hilton MF, Mantzoros CS, and Shea SA. Adverse metabolic and cardiovascular consequences of circadian misalignment. Proc Natl Acad Sci U S A 106: 4453-4458, 2009.

20. Stokkan K-A, Yamazaki S, Tei H, Sakaki Y, and Menaker M. Entrainment of the Circadian Clock in the Liver by Feeding. Science 291: 490-493, 2001. 
21. Stratmann M and Schibler U. Properties, Entrainment, and Physiological Functions of Mammalian Peripheral Oscillators. J Biol Rhythms 21: 494-506, 2006.

22. Wang Q, Brunner HR, and Burnier M. Determination of cardiac contractility in awake unsedated mice with a fluid-filled catheter. Am J Physiol Heart Circ Physiol 286: H806-814, 2004.

23. Wang Q, Horisberger J-D, Maillard M, Brunner HR, Rossier BC, and Burnier M. Salt- And Angiotensin II-Dependent Variations In Amiloride-Sensitive Rectal Potential Difference In Mice. Clin Exp Pharmacol Physiol 27: 60-66, 2000.

24. Wang Q, Hummler E, Nussberger J, Clement S, Gabbiani G, Brunner HR, and Burnier M. Blood Pressure, Cardiac, and Renal Responses to Salt and Deoxycorticosterone Acetate in Mice: Role of Renin Genes. J Am Soc Nephrol 13: 1509-1516, 2002.

25. Woon PY, Kaisaki PJ, Bragança J, Bihoreau M-T, Levy JC, Farrall M, and Gauguier D. Aryl hydrocarbon receptor nuclear translocator-like (BMAL1) is associated with susceptibility to hypertension and type 2 diabetes. Proc Natl Acad Sci U S A 104: 14412-14417, 2007.

26. Young ME. The circadian clock within the heart: potential influence on myocardial gene expression, metabolism, and function. Am J Physiol Heart Circ Physiol 290: H1-16, 2006.

27. Young ME and Bray MS. Potential role for peripheral circadian clock dyssynchrony in the pathogenesis of cardiovascular dysfunction. Sleep Med 8: 656-667, 2007.

28. Zuber AM, Centeno G, Pradervand S, Nikolaeva S, Maquelin L, Cardinaux Lo, Bonny O, and Firsov D. Molecular clock is involved in predictive circadian adjustment of renal function. Proc Natl Acad Sci U S A 106: 16523-16528, 2009. 


\section{Figure Legends}

Figure 1. Effect of PAR bZip gene disruption on cardiac function

A) Heart weight to body weight ratio (HW/BW) as an index of cardiac hypertrophy in PAR bZip KO (black), HZ (grey) and WT (white) mice. $\mathrm{n}=14-16$ mice per group.

B) Lung weight to body weight ratio (LW/BW) an index of heart failure. $n=8$ mice per group.

C) Left ventricular end diastolic pressure (LVEDP), a sign of cardiac dysfunction, is significantly elevated in the KO.

D) Time constant of isovolumic relaxation (Weiss Tau), an index of cardiac relaxation time, is significantly prolonged in the KO.

E) $\mathrm{LVdP} / \mathrm{dt}_{\min }$ and $\mathrm{LVdP} / \mathrm{dt}_{\max }$ assessing left ventricular contractility, systolic and diastolic blood pressure, respectively, are significantly decreased in both $\mathrm{KO}$ and $\mathrm{HZ}$ mice.

F) Left ventricular systolic pressure (LVSP) is markedly decreased in the KO mice.

$\mathrm{n}=6-8$ mice per group for hemodynamic measurements.

Values are means $\pm \mathrm{SEM}, * \mathrm{p} \leq 0.05, * * \mathrm{p} \leq 0.01 \mathrm{KO}$ vs. WT, and HZ. $\# \mathrm{p} \leq 0.05 \mathrm{HZ}$ vs WT, one-way ANOVA.

Figure 2. Influence of the deletion of the PAR bZip genes on daily mean blood pressure (MBP) and heart rate (HR).

A) Individual tracing of MBP and HR in PAR bZip knockout (KO), heterozygous (HZ), and wild-type (WT) mice during 48 hours.

B) 24-hour telemetric MBP and HR in PAR bZip KO (ם), HZ ( $\triangle$ ) and WT ( $\square)$ mice. Each time point represents the average of 3 hours of monitored data. MBP and HR in the KO mice have the same circadian rhythm as WT and HZ. However, MBP of KO is significantly lower (upper panel) and HR higher (lower panel) over the course of the day compared to the WT. 
HZ have an intermediate phenotype. X-axis, black Box indicates the dark period and white box indicates the light period. Values are means \pm S.E.M., $\mathrm{n}=5$ mice per group. ${ }^{*} p \leq 0.05, * *$ $p \leq 0.01, \mathrm{KO}$ vs. WT.

Figure 3: Exacerbated response to $\beta_{1}$-receptor blockade in PAR bZip KO mice

A) HR response (expressed at the \% of difference of HR) to atenolol in PAR bZip KO mice $(\square), H Z(\triangle)$ and WT ( $\square)$ mice.

B) Difference in left ventricular systolic pressure response induced by atenolol injection.

$\mathrm{C}$ and D) Changes in LVdP/dtmax and LVdP/dtmin in response to atenolol.

Values are mean $\pm \mathrm{SEM}, \mathrm{n}=5$ mice per group. $* p \leq 0.05, * * p \leq 0.01$, one-way ANOVA.

Figure 4. Influence of the deletion of the PAR bZip genes on sodium and fluid intake, and on urinary electrolyte excretion.

A) Influence of PAR bZip gene knockout on urinary volumes in mice. Urine was collected in the morning (ZT0-ZT4) and in the night (ZT12-ZT16), respectively.

B) 24-hour recordings of water intake.

C) 24-hour recordings of sodium intake. Values are based on the intake of food containing 3 $\operatorname{mg~Na}{ }^{+} / \mathrm{g}$.

D) Urinary creatinine excretion in $\mathrm{KO}, \mathrm{HZ}$, and WT mice.

E) Urinary sodium excretion expressed as urinary sodium to creatinine ratio.

F) Urinary potassium excretion expressed as urinary sodium to creatinine ratio.

Values are means $\pm \mathrm{SEM}, \mathrm{n}=10$ mice per group. ${ }^{*} p \leq 0.05, * * p \leq 0.01, * * * p \leq 0.001 \mathrm{KO}$ vs. HZ and WT, one-way ANOVA. X-axis, black boxes indicates the dark period (from 19:00 to 23:00 or ZT 12-16), and white boxes indicates the light period (ZT0-ZT4). 
Figure 5. Response to aldosterone and aldosterone synthesis in PAR bZip KO mice

A) Response of ENaC activity to exogenous aldosterone. The rectal $\triangle \mathrm{PD}$ amil was measured before and 6 hours after the administration of aldosterone $(6 \mathrm{mg} / \mathrm{kg}$, i.p). No significant difference is observed between $\mathrm{KO}$ and WT. Values are means $\pm \mathrm{SEM}, \mathrm{n}=6$ per group.

B). Plasma aldosterone levels in PAR bZip KO (black), HZ (grey) and WT (white) mice. Plasma aldosterone levels are significant lower in the KO mice. Values are means $\pm \mathrm{SEM}$, $\mathrm{n}=6-8$ mice per group, ${ }^{*} p \leq 0.05 \mathrm{KO}$ vs. HZ or vs. WT, one-way ANOVA.

Figure 6. Activity of the epithelial sodium channel $(\mathrm{ENaC})$ in distal colon.

The amiloride-sensitive potential difference (ami-s-PD or $\triangle$ PDamil) in colon as an index of $\mathrm{ENaC}$ activity was measured daily in the morning and the night for 3 days. The amplitude of circadian oscillations of ami-s-PD are comparable in WT ( $\square)$ and HZ $(\Delta)$, but completely abolished in the KO ( $\mathbf{})$. Values are means \pm SEM, $n=6-8$ per group. ${ }^{*} p \leq 0.001 \mathrm{KO}$ vs. $\mathrm{HZ}$ and WT, one-way ANOVA.

X-axis, black boxes indicates the dark period (from 19:00 to 23:00 or ZT 12-16), and white boxes indicates the light period (ZT0-ZT4). 
Figure 1
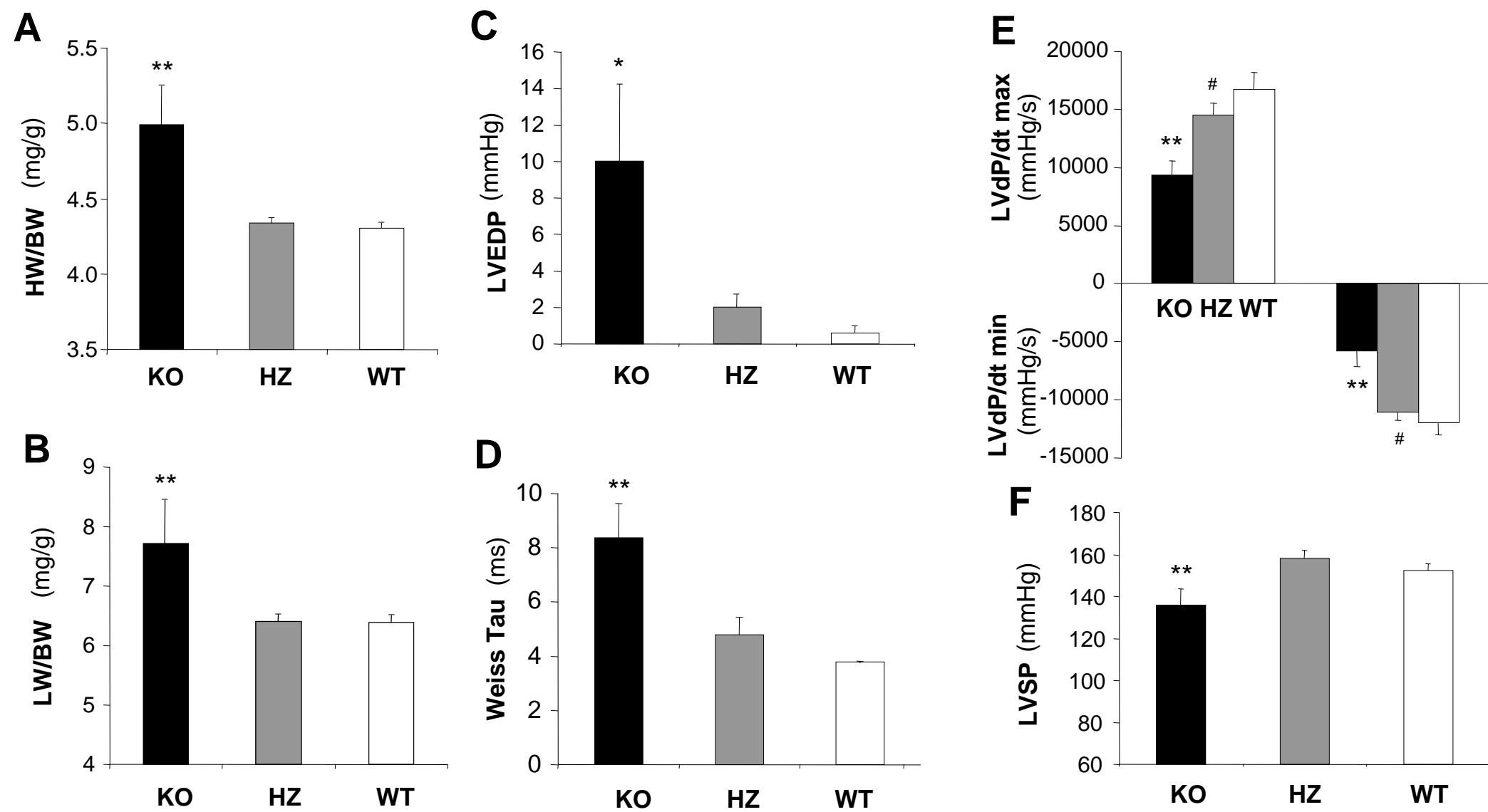


\section{Figure 2}

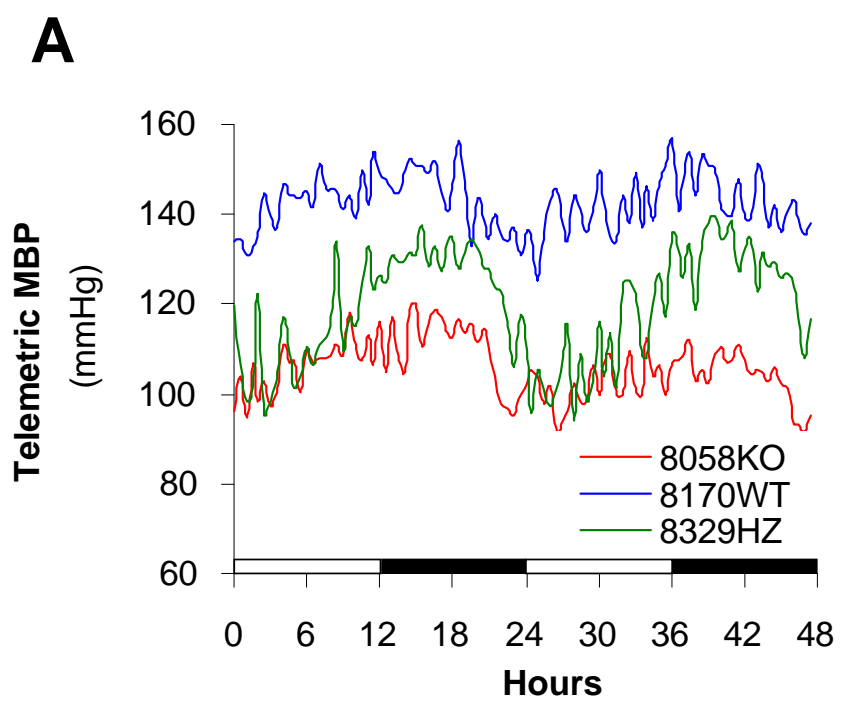

B
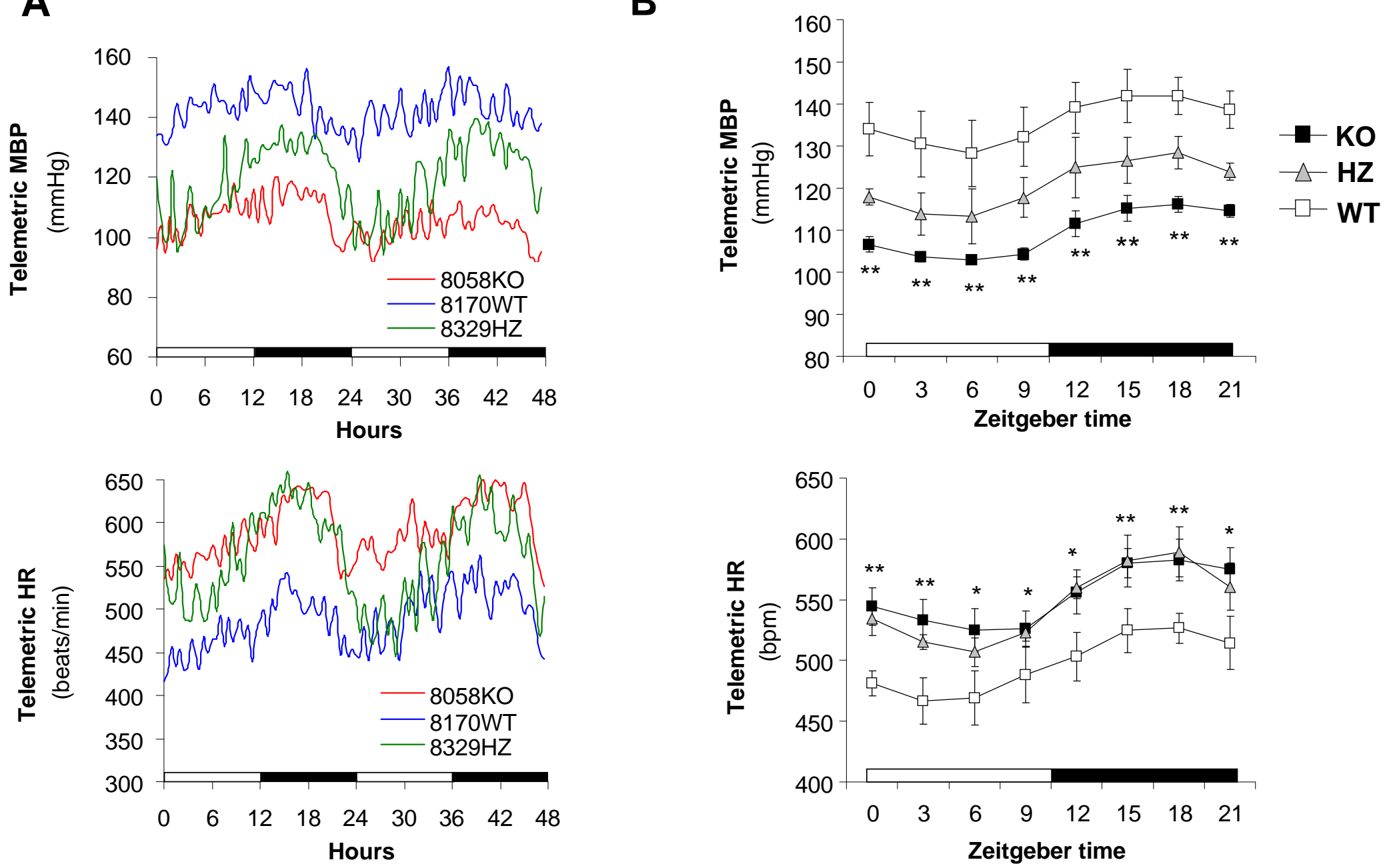
Figure 3
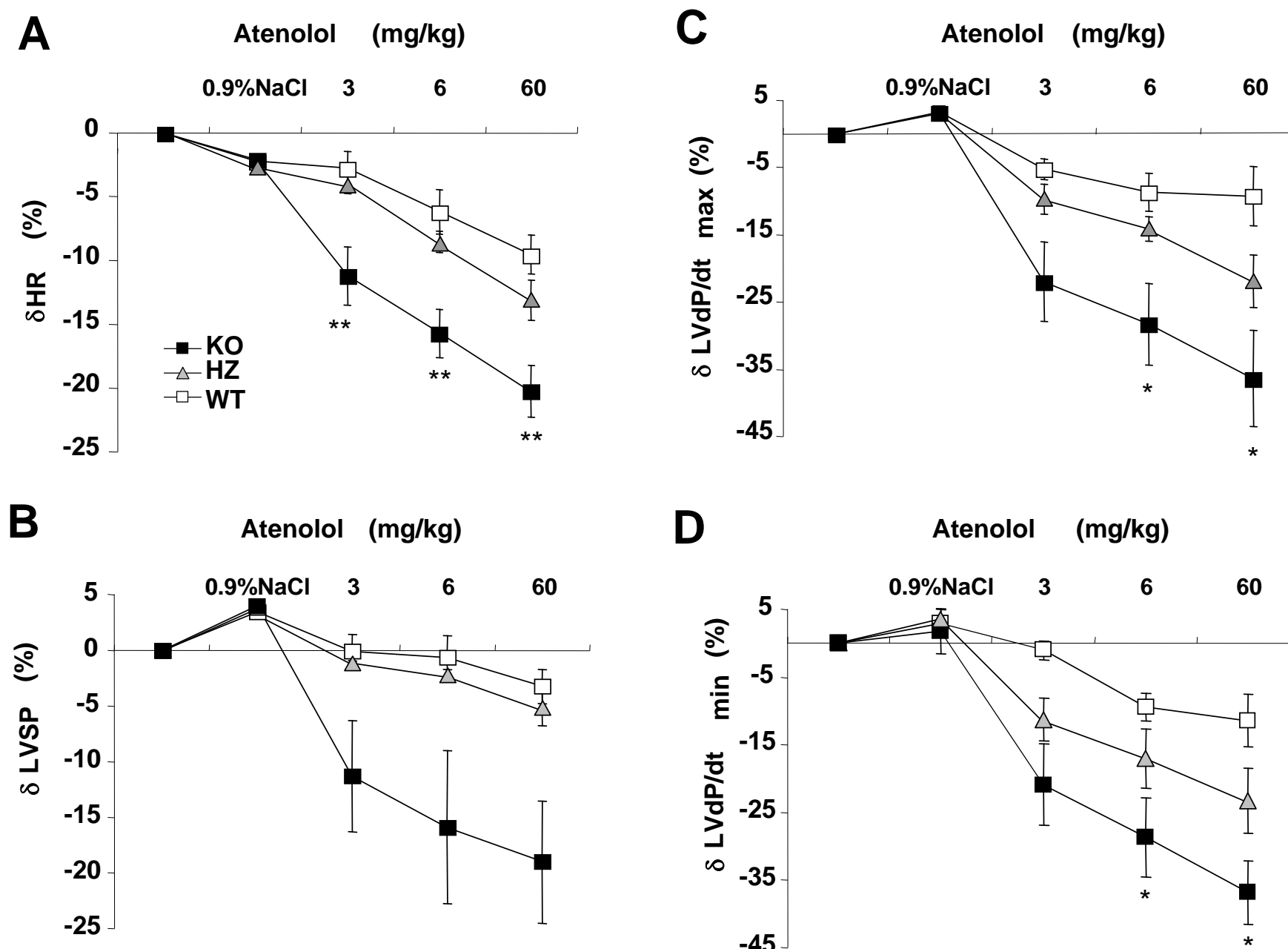

D Atenolol (mg/kg)

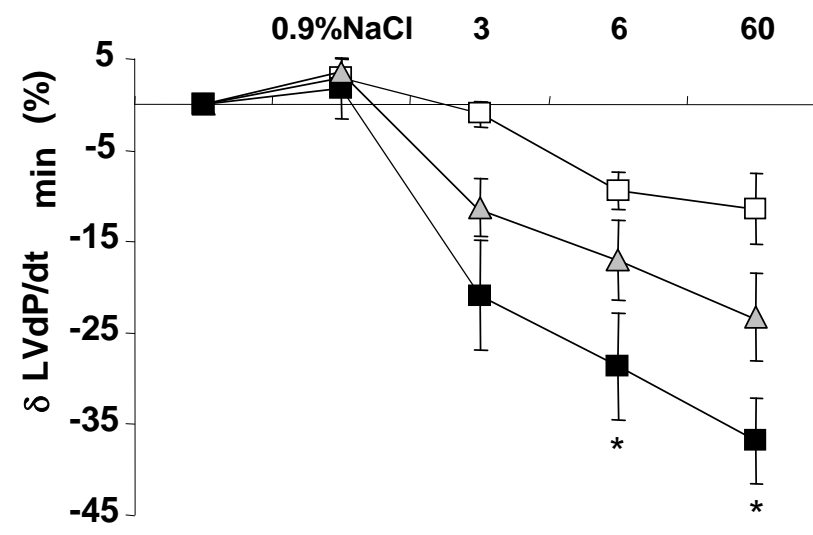




\section{Figure 4}

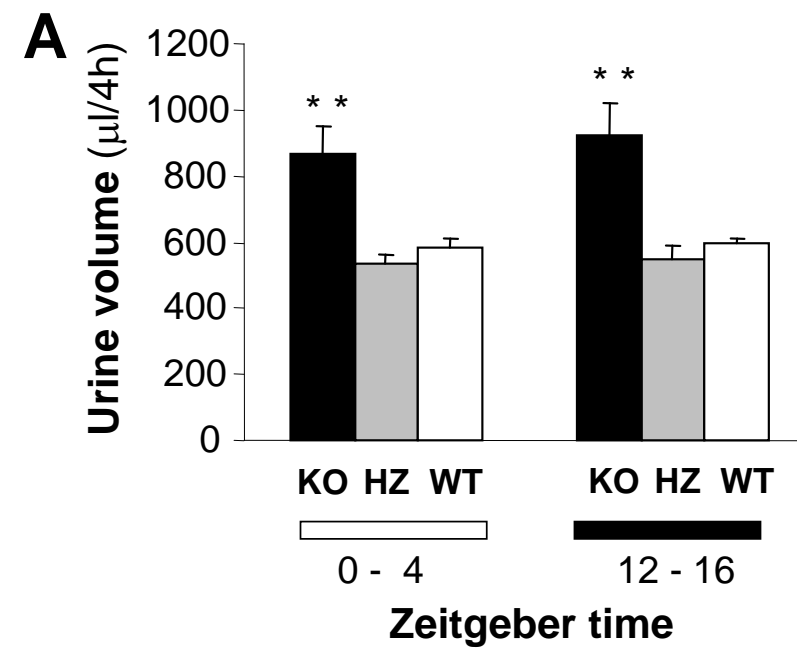

B

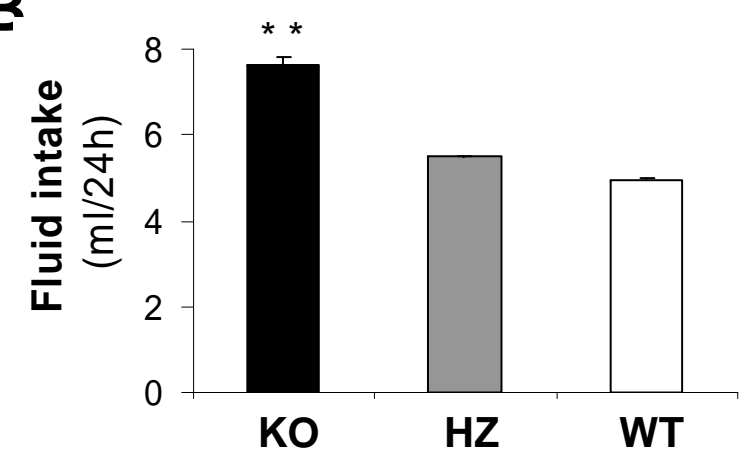

C

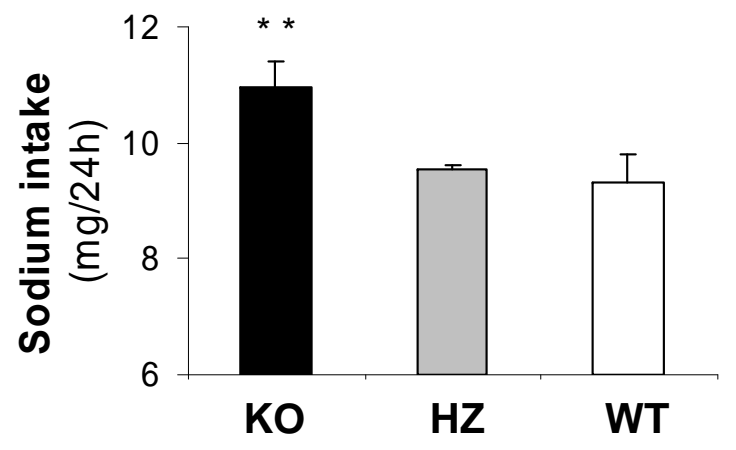

D

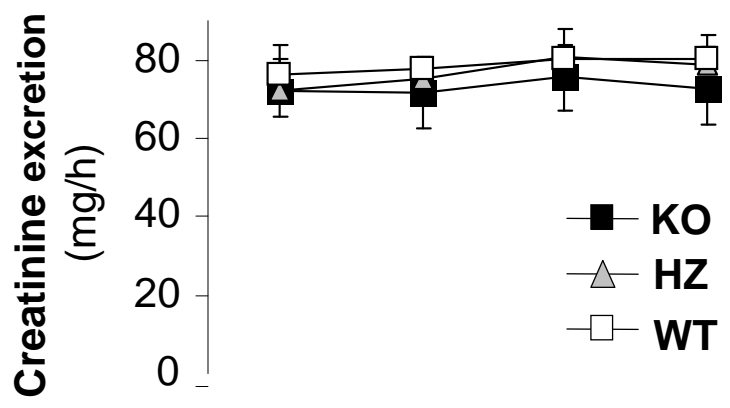

E

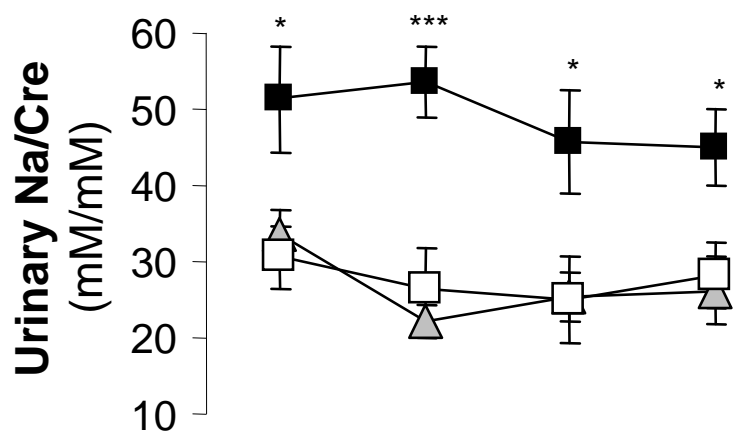

F

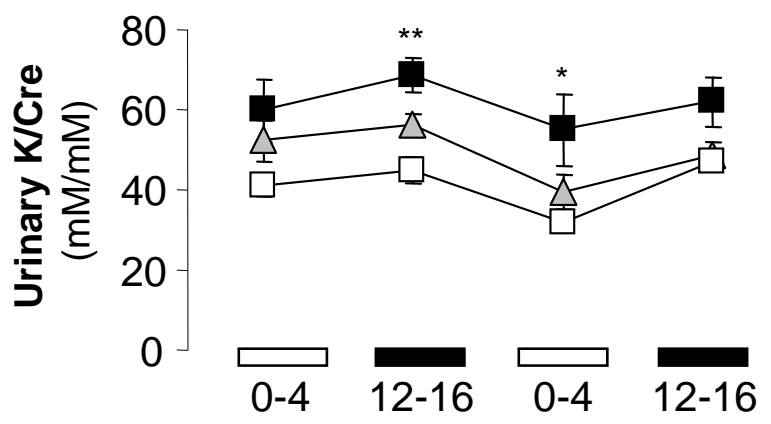

Zeitgeber Time 
Figure 5

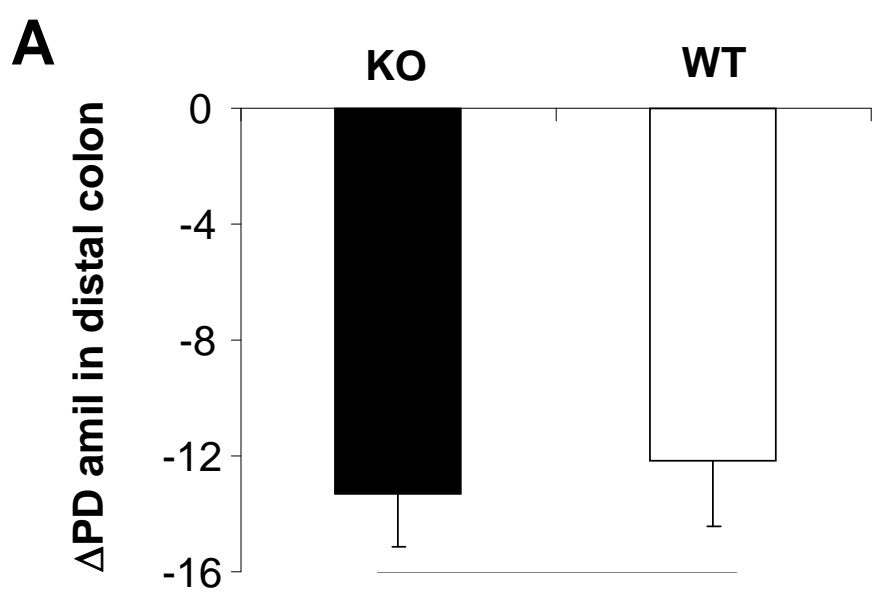

B

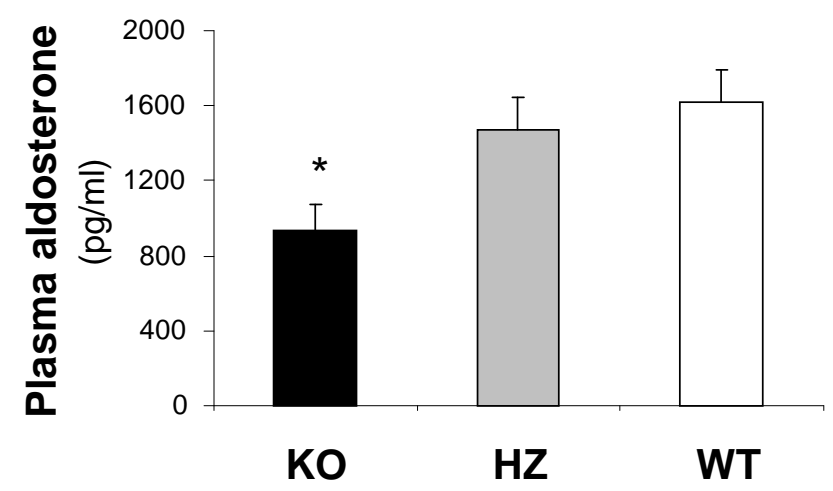


Figure 6

\section{Zeitgeber Time}

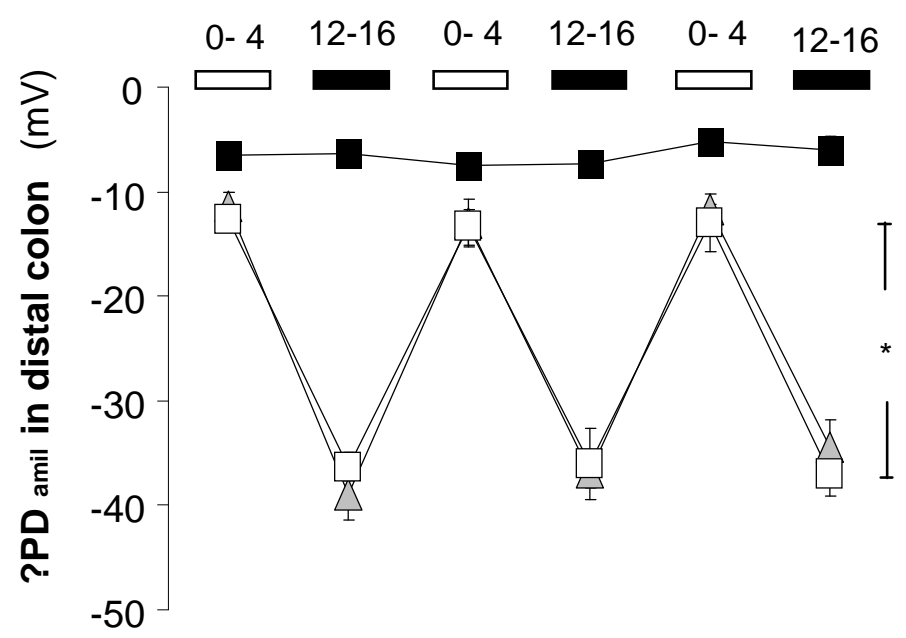

